\title{
Behind Recurrent Thromboembolic Events
}

\author{
Sara Guerreiro ${ }^{1}$, Carolina Gouveia² ${ }^{2}$ Rita Mora de Féria ${ }^{3}$, Ana Lourenço², Fátima Grenho² \\ ${ }^{1}$ Hospital de Santa Cruz, Centro Hospitalar Lisboa Ocidental, Lisbon, Portugal \\ ${ }^{2}$ Hospital São Francisco Xavier, Centro Hospitalar Lisboa Ocidental, Lisbon, Portugal \\ ${ }^{3}$ Hospital Prof. Dr. Fernando da Fonseca, Lisbon, Portugal
}

Received: 21/02/2016

Accepted: 31/05/2016

Published: 03/08/2016

How to cite this article: Guerreiro S, Gouveia C, Mora de Féria R, Lourenço A, Grenho F. Behind recurrent thromboemobolic events. EJCRIM 2016;3:doi:10. 12890/2016_000413.

Conflicts of Interests: The Authors declare that there are no competing interests.

This article is licensed under a Commons Attribution Non-Commercial 4.0 License

\section{ABSTRACT}

We describe the case of a 68-year-old man, who presented with an ischemic stroke due to cardiac embolization related to mitral valve endocarditis. Blood cultures were always negative and post-operative valve histology did not show microorganisms. The patient also presented further recurrent peripheral embolic events. These clinical aspects were the first sign of a pancreas adenocarcinoma, which was only diagnosed in the clinical autopsy. In conclusion, these clinical findings of recurrent thromboembolic events with no microorganisms isolated suggests the diagnostic of a marantic endocarditis.

\section{LEARNING POINTS}

- Marantic endocarditis is characterized by the deposition of fibrin and thrombi on heart valves in the absence of microorganisms.

- Is associated with higher incidence of thromboembolic events and most commonly found in patients with neoplasia in post-mortem studies.

- It is a diagnostic challenge to distinguish from infective endocarditis and a high clinical suspicion is crucial to confirm the diagnosis.

\section{KEYWORDS}

Marantic endocarditis; thromboembolism; pancreatic adenocarcinoma

\section{CASE REPORT}

A 68-year-old man was admitted to the emergency department of our hospital with 1-hour evolution of left hemiparesis and hypoesthesia, left facial paralysis and mild dysarthria. Cranial Computed Tomography (CT) showed aspects suggesting right hemispheric acute ischemic stroke. The initial laboratory blood tests revealed a platelet count of $74.000 / \mathrm{mm}^{3}$, which contraindicated the thrombolytic treatment. The other lab results were within normal ranges values. Chest $X$-ray and 12-lead electrocardiography findings were normal.

The patient's medical history included hypertension, chronic gastritis and small hiatus hernia; he was a former smoker, with moderate alcoholic habits.

To clarify the aetiology of stroke, a transthoracic echocardiography (TTE) was done and revealed a mobile and echodense lesion on the atrial surface of posterior mitral valve leaflet suggesting a vegetation. The transesophageal echocardiography (TEE) showed the vegetation with $20 \mathrm{~mm}$ of major diameter, mild mitral regurgitation and no leaflet destruction (Fig. 1). As such, the diagnostic of infective endocarditis was admitted and empiric antibiotic therapy was initiated, with gentamicin and vancomycin. Serial blood cultures remained sterile, despite the extension of incubation time to 21 days. Serologic tests for atypical microorganisms were negative. The immunologic evaluation was unremarkable. 
The TEE was repeated after 15 days of antibiotic treatment and a growth of vegetation was seen, with a large and mobile vegetation attached on posterior and anterior mitral valve leaflets and associated with moderate mitral regurgitation. TEE also showed a suspicion of anterior leaflet rupture. Regarding hypothetical fungi infection, we decided to add to treatment amphotericin B due to vegetation morphologic aspects. The following day, the patient developed signs of acute upper limb ischemia and underwent vascular surgery (thromboembolectomy). The bacterial and mycology exam of thrombus was negative.

At the time, we considered uncontrolled infection with two embolic events and enlarging vegetation, despite the large spectrum antibiotic. The patient underwent cardiac surgery with replacement of the mitral valve. The microbiologic test of the valve was negative and histopathologic examination revealed vegetation constituted by thrombus. These clinical and pathologic findings suggested non-infective thrombotic endocarditis. During the post-operative period (on the 4th day), the patient experienced further thrombotic events, with irreversible left lower limb ischemic and distal right foot ischemic. Additionally, there was a clinical decline of hemodynamic instability, respiratory and acute renal failure. TEE was performed and showed no prosthesis alterations, including no vegetation. Meanwhile, the transaminases raise and cholestasis pattern were observed and elevated plasma levels of CA-125 at $2090 \mathrm{U} / \mathrm{mL}$ (normal < 35), CA 15.3 at $202 \mathrm{U} / \mathrm{mL}$ (normal < 31) and CYFRA 21 at 88,9 (<2.1) were detected. Abdominal ultrasonography revealed heterogeneous liver with several hipoechogenic images, suggesting infarction or secondary deposits. The patient's clinical and hemodynamic status worsened and he died on 9 th day post- operation. The clinical autopsy revealed a poorly differentiated ductal adenocarcinoma in the tail of the pancreas, measuring 8 centimetres. Other relevant findings included: splenic artery invasion by the adenocarcinoma of the pancreas; metastasis in the liver, pleura and lungs; and thromboembolic infarctions in multiple organs, such as the kidneys, lungs, brain and heart (Fig. 2).
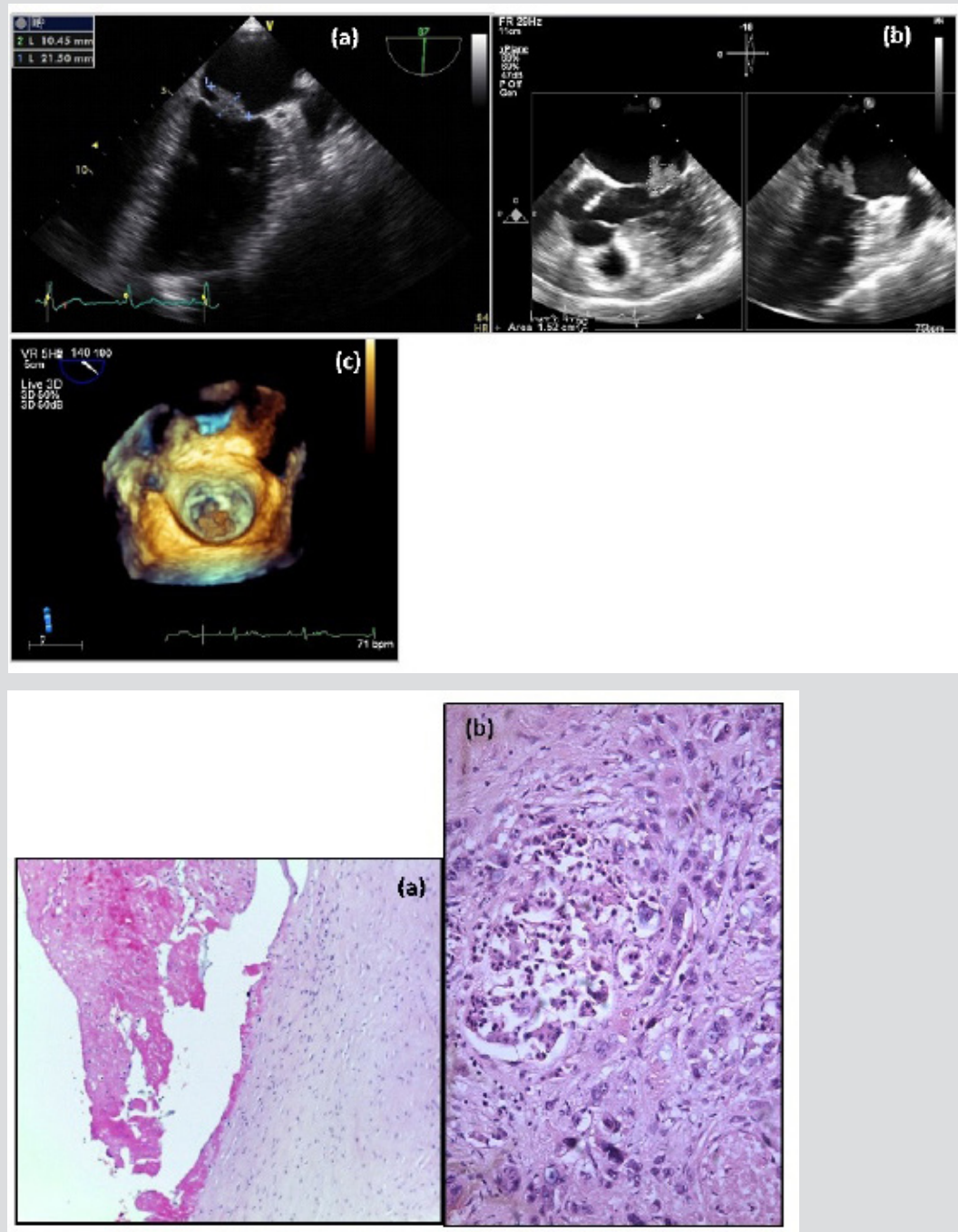

Figure 1. Transesophageal echocardiography showing vegetation on the mitral valve.

(a) Apical 4 chamber view on first TEE showing hypoechoic and homogenous structure on anterior mitral leaflet, although no destruction of the leaflet was observed. (b) Apical 3 chambers view on second TEE showing enlarging of vegetation, which was attached to the anterior and posterior mitral leaflets.

(c) 3D TEE with atrial face of mitral valve view showing the vegetation.

\section{Figure 2. Clinical autopsy.}

(a) Marantic endocarditis demonstrated by an aseptic thrombus on the left, loosely attached to the mitral valve leaflet on the right.

(b) Poorly differentiated ductal adenocarcinoma of the pancreas invading an islet of Langerhans. 


\section{DISCUSSION}

Marantic endocarditis (also known as non-bacterial thrombotic endocarditis) is a rare condition, characterized by the deposition of fibrin and platelets on heart valves in the absence of a bloodstream bacterial infection. There is a well-established association between patients with neoplasia and higher rates of marantic endocarditis, most often found in autopsy ${ }^{[1,2]}$. Patients with adenocarcinoma were at a higher risk than patients with other malignant processes, especially in cases of pancreatic cancer ${ }^{[3]}$. The pathogenesis is unclear, with a multifactorial mechanism behind the hypercoagulability state ${ }^{[4]}$.

As this case report suggests, the diagnosis of marantic endocarditis can be difficult to establish ante mortem because of the paucity of symptoms and can often be confused with culture-negatives infective endocarditis.

Thus, a high index of clinical suspicion is critical in these patients. Compared to infective endocarditis, there is a greater tendency for vegetation to embolize and cause extensive infarction. In this particular case, the patient did not have any past history of neoplasia suspicion and general analysis, including liver function tests at the beginning of hospitalization, were normal. However, pancreatic tumour is silent, the symptoms appear often in advanced stages and the mucinous histologic type is strongly associated with embolism presentation.

A few comments should be made about how the diagnostic investigation was conducted in this case. First of all, we should not have assumed the infective endocarditis as the definitive diagnosis because the serial blood cultures were negative as were the serologic tests for atypical microorganisms. Thus, we should have researched other causes of recurrent arterial thromboembolic events. The strong and recognized association between cancer and thrombophilia induces a high suspicion of occult neoplasia in this patient.

Second, other exams could have been performed before the patient underwent cardiac surgery. The initial work-up of an unknown primary cancer includes an exhaustive physical examination, blood analysis and a CT scan of the chest, abdomen and pelvis with intravenous contrast. This imaging test would have been crucial for the diagnosis of pancreatic cancer in a patient with non-infective endocarditis and recurrent thromboembolic events.

Tumor markers were requested after surgery, when the liver tests started to change and some of them were elevated. However, the majority of tumor markers have low specificity with a higher percentage of false positives and, for this reason, their use for screening is not recommended. They can be used to monitor and establish prognosis in patients with already confirmed cancer.

It is clear that this extended analysis should not have been performed routinely in all patients with stroke but only in patients with poorly defined aetiology, and especially in those with non-bacterial endocarditis and new embolic events.

Finally, it is important to highlight that more routine investigation should have been done to be sure of the diagnosis before refering the patient to an invasive procedure, such as cardiac surgery.

This case brings forward the importance of the clinician, who no longer presumes that a patient has infective endocarditis but, instead, carries out more extensive investigations to look for a potential cause for non-bacterial thrombotic endocarditis.

\section{REFERENCES}

1. Llenas-García J, Guerra-Vales JM, Montes-Moreno S, et al. Nonbacterial Thrombotic Endocarditis: clinicopathologic study of a necropsy series. Rev Esp Cardio 2007;60:493500 .

2. Biller J, Challa VR, Toole JF et al. Nonbacterial thrombotic endocarditis. A neurologic perpective of clicopathologic correlations of 99 patients. Arch Neurol 1982;39:95-8.

3. El-Shami K, Griffiths E, Streiff M. Nonbacterial thrombotic endocarditis in cancer patients: pathogenesis, diagnosis, and treatment. Oncologist 2007;12:518-23.

4. Mazokopakis EE, Syros PK, Starakis IK. Nonbacterial Thrombotic Endocarditis (Marantic Endocarditis) in Cancer Patients. Cardiovasc Hematol Disord Drug Targets 2010;10:84-6. 